\title{
New Possibilities of Diagnostics of Non-Alcoholic Fatty Liver Disease
}

\author{
Pirogova I Yu ${ }^{1,2} *$, Yakovleva $S^{2}$, Neuimina Tatyana Valeryevna ${ }^{1,3}$, Sinitsyn SP $^{2,4}$, Chulkov VS ${ }^{2,5}$ and Shamaeva \\ Tatyana Nikolaevna ${ }^{6}$ \\ ${ }^{1}$ Doctor of Medicine, Associate Professor of the Department of Faculty Therapy of the State Educational Institution, South Ural State Medical \\ University, Head of the Center for Gastroenterology and Hepatology, LLC Medical Center "Lotos", Chelyabinsk, Russia
}

${ }^{2}$ Head of the Department of Therapy LLC Medical Center "Lotos", Russia

${ }^{3}$ Doctor-gastroenterologist 000 Medical center "Lotos", Russia

${ }^{4}$ Doctor of medical sciences, professor, Head of the Department of Faculty Therapy of the State Educational Institution, South Ural State Medical University, Russia

${ }^{5} \mathrm{MD}$, Associate professor of the Department of Faculty Therapy of the State Educational Institution, South Ural State Medical University, Russia

${ }^{6}$ Associate Professor of the Department of Mathematics, Medical Informatics, Informatics and Statistics, Physics of the State Educational Institution, South Ural State Medical University, Russia

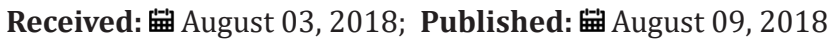

*Corresponding author: Pirogova I Yu, Doctor of Medicine, Associate Professor of the Department of Faculty Therapy of the State Educational Institution, South Ural State Medical University, Head of the Center for Gastroenterology and Hepatology, LLC Medical Center "Lotos", Chelyabinsk, Russia

\section{Abstract}

The aim of the study was to create a non-invasive model for diagnosing fibrosis and steatosis in non-alcoholic fatty liver disease. Materials and methods. The study included 183 patients with liver steatosis according to ultrasound of the liver. General clinical examination, verification of the stage of fibrosis and steatosis of the liver on the Fibroscan 502 TOUCH device with the CAP software was carried out. Results of the study. Fibrosteatotest (c), based on stigmas of inflammation activity, insulin resistance, lipid spectrum for the diagnosis of fibrosis and steatosis (diagnostic accuracy of more than $80 \%$ compared to the results of fibroscanning), is created by the method of discriminant analysis, which allows to identify patients with the 2nd and more stage of fibrosis and steatosis of the liver, who need medication.

Conclusions: The Fibrosteatotest (c) test allows for screening patients to identify the stages of fibrosis and steatosis of the liver that require drug therapy.

Keywords: Non-Alcoholic Fatty Liver Disease; Fibrosis and Steatosis of the Liver; Fibrosteatotest

\section{Introduction}

Non-alcoholic fatty liver disease (NAJBP) has various clinical forms - from steatosis of the liver and steatohepatitis to liver cirrhosis and hepatocellular carcinoma-differing in severity, spectrum of complications, and disease prognosis. With the progression of NAJBP (the growth of the fibrosis stage), the risk of cardiovascular diseases, type 2 diabetes mellitus (DM-II) increases in parallel with the risk of progression of hepatic insufficiency. Today, NAJIB is regarded as a hepatic component of metabolic syndrome (MS). The treatment of patients suffering from NUZHBP provides early diagnosis and treatment of concomitant metabolic diseases in order to prevent the progression of hepatic insufficiency and metabolic complications [1]. Undoubtedly, the first step in the treatment of patients with NAJBP should be considered a lifestyle modification. Pharmacotherapy should be given to patients with moderate and severe fibrosis ( $>$ F2). Early detection of patients with NADD with severe liver fibrosis is relevant for clinical practice. Given the lack of clinical indications for liver biopsy in this category of patients, it is necessary to develop and widely implement noninvasive methods for diagnosis of fibrosis and steatosis of the liver in NAZHBP both for primary diagnosis and for evaluation of treatment outcomes. Treatment is also indicated for patients with 
less severe fibrosis stage, but with a high risk of its progression (with CD-II, MS, sustained increase in ALT and necroinflammatory reaction in liver tissue) [2].

\section{Objective}

To study the markers of inflammatory activity, insulin resistance, lipid spectrum and their contribution to the formation of fibrosis and steatosis of the liver, non-invasive evaluation of fibrosis and fibroscan stage (FbroScan 502 TOUCH with CAP software), and development of a noninvasive method for diagnosis of fibrosis and steatosis liver in patients with NAJBP for early diagnosis of the disease based on clinical and laboratory data, comparison with the results of serum fibrosis evaluation tests (GUCI, APRI, Fib4, Forns, MDA - selection.

\section{Materials and Methods}

In the study, 183 patients with a BMI> $25 \mathrm{~kg} / \mathrm{sq}$. M, steatosis of the liver according to ultrasound of the liver. Male 101, female 82, median age 44 (38-49) years. All patients underwent a clinical examination including assessment of clinical syndromes and quality of life (SF-36 questionnaire), elimination of alcoholic liver damage according to the AUDIT questionnaire (score less than 8 points), lipid spectrum, carbohydrate metabolism, insulin resistance, inflammatory activity in the liver level of ALT, AST, CRP) and cholestasis syndrome. In addition, autoimmune, cholestatic liver diseases, drug-induced hepatitis and Wilson-Konovalov's disease are excluded. The data obtained during the examination of patients allowed to assess the presence of liver fibrosis using the NAFLD fibrosis score. Evaluation of the stage of fibrosis and the degree of steatosis was carried out on the Fibroscan 502 TOUCH apparatus with an ultrasonic sensor for obese patients with the CAP software. The technique allows simultaneous non-invasive determination of the stage of fibrosis and steatosis at the point of the study. The sensitivity and specificity of the method is about 90\% (compared with liver biopsy) [1]. Statistical analysis of the results was carried out using the software package for statistical analysis of IBM SPSS Statistics 19.

\section{Results of the Study}

The general characteristics of a group of patients are shown in Table 1. Patients who were included in the group, mostly men, middle-aged, had an excess of body weight or obesity - the median BMI was $30 \mathrm{~kg} / \mathrm{sq}$. M. Two-thirds of the patients had MS components in the first line of kinship, more than half of them reported eating less than 3 times a day (lack of breakfast, late supper) and inactivity. Violation of carbohydrate metabolismSD-II or NGN-was observed in $60 \%$ of patients. Two thirds of patients had hypertensive dyslipidemia (hypercholesterolemia, hypertriglyceridemia), pathology of the biliary tract (according to the US - cholecystitis in $53 \%$ and CLS in $11 \%$ of cases). Two or more components of the metabolic syndrome had two-thirds of the patients. Fibroscanning of the liver was performed for all patients with a diagnostic purpose, in Further it defined or determined medical tactics-purpose or appointment of medicamental therapy in addition to recommendations on modification of a way of life. The average value of the elasticity of the liver was $\mathrm{Me}=7.5 \mathrm{kPa}$, which corresponds to the 2 stages of liver fibrosis. The stage of steatosis $-\mathrm{Me}=310 \mathrm{kPa}$ - in this case corresponded to 2-3 stages.

Table 1: General characteristics of a group of patients $(n=183)$.

\begin{tabular}{|c|c|}
\hline Parameter & Absolute quantity /\% \\
\hline Men $101(55 \%)$ & Men $101(55 \%)$ \\
\hline Women 82 (45\%) & Women $82(45 \%)$ \\
\hline Age (years) (Me (25 - $75 \mathrm{P})$ & $44(38-49)$ \\
\hline BMI kg / sq. M (Me (25 - 75 P) & $30(28-34)$ \\
\hline Waist circumference, cm (Me (25 - 75 P) & $97(92-104)$ \\
\hline $\begin{array}{l}\text { Components of the metabolic syndrome } \\
\text { in the first line of kinship }\end{array}$ & $112(61 \%)$ \\
\hline Hypodinamy & $142(77 \%)$ \\
\hline $\begin{array}{c}\text { Eating less than } 3 \text { times a day (no } \\
\text { breakfast) }\end{array}$ & $118(64 \%)$ \\
\hline Diabetes & $18(14 \%)$ \\
\hline Impaired fasting glycemia (NGN) & $54(42 \%)$ \\
\hline Hypertonic disease & $118(64 \%)$ \\
\hline Dyslipidaemia & $116(63 \%)$ \\
\hline Cholecystitis (according to ultrasound) & $98(53 \%)$ \\
\hline LCM (according to ultrasound) & $20(11 \%)$ \\
\hline Parameter & Result (Me (25 - 75 P) \\
\hline $\begin{array}{l}\text { ALT, the norm is up to } 40 \text { units / } 1 \text { (Me } \\
\qquad(25-75 \mathrm{P})\end{array}$ & $69(62-77)$ \\
\hline $\begin{array}{l}\text { AST, the norm is up to } 40 \text { units / liter } \\
(\mathrm{Me}(25-75 \mathrm{P})\end{array}$ & $73(68-79)$ \\
\hline $\begin{array}{l}\text { GGT, the norm is up to } 50 \text { units / } 1 \text { (Me } \\
\qquad(25-75 \mathrm{P})\end{array}$ & $76(41 \%)$ \\
\hline $\begin{array}{l}\text { CRP, the norm is up to } 3 \text { units (Me (25 } \\
\qquad-75 \mathrm{P})\end{array}$ & $4,6(4,1-5,0)$ \\
\hline $\begin{array}{l}\text { Glycemia, the norm to } 6 \mathrm{mmol} / \mathrm{l}(\mathrm{Me} \\
\qquad(25-75 \mathrm{P})\end{array}$ & $6,0(5,6-6,4)$ \\
\hline HOMA-index, norm up to 2,7 & $3,7(3,4-4,2)$ \\
\hline Cholesterol, mmol / l, (Me (25 - 75 P) & $6,3(5,8-6,7)$ \\
\hline Triglycerides mmol / 1 (Me (25 - 75 P) & $2,1(1,8-2,4)$ \\
\hline LDL, mmol / L, (Me (25 - 75 P & $3,8(3,4-4,2)$ \\
\hline HDL mmol / L, (Me (25 - 75 P) & $1,2(0,8-1,4)$ \\
\hline The coefficient of atherogenicity & $3,9(3,5-4,3)$ \\
\hline \multicolumn{2}{|l|}{ Fibro scanning of the liver: } \\
\hline $\begin{array}{l}\text { Elasticity-liver fibrosis-normal up to } \\
5.8 \mathrm{kPa}\end{array}$ & $7,5(7,1-7,9)$ \\
\hline Steatosis of the liver, norm up to $200 \mathrm{~dB}$ & $310(286-342)$ \\
\hline
\end{tabular}

Given that liver biopsy in NAJB is rarely performed in a wide clinical practice due to the lack of medical indications, and Fibroscan 502 TOUCH with the CAA software remains inaccessible, it is interesting to develop a noninvasive method for diagnosing both fibrosis and steatosis of the liver based on clinical and laboratory data, replacing liver fibroscans. To determine the significance of the studied parameters in the formation of fibrosis and steatosis 
of the liver, a correlation analysis was performed (Table 2). As can be seen from the data of the table, the stage of fibrosis and steatosis of the liver in NAJBP correlates with the stigma of the disease activity, insulin resistance, dyslipidemia, the presence of metabolic syndrome components. Models of diagnostics of fibrosis and steatosis of the liver are presented in the form of discriminant analysis (Table 3) and 4. The equations of the discriminant functions (Figures $1 \& 2$ ) with high sensitivity and specificity can diagnose fibrosis and steatosis of the liver of the second and more stages (Fibrosteatotest (C) standard methods of research available in a wide clinical practice. For the standard of diagnosis of the stage of fibrosis and steatosis of the liver, the results of fibroscanization of the liver of the device Fibroscan 502 with the software of the CAPS were taken. The created method of non-invasive diagnostics of liver steatosis can, therefore, with a diagnostic accuracy of more than $80 \%$, replace the diagnosis of fibrosis and steatosis of the liver with this fibroscan model. The diagnostic model for fibrosis Fibrosteatotest (C) is characterized by less sensitivity and specificity, as for a more accurate diagnosis of fibrosis, direct serum markers are required. The use of these reagents is often not available, so Fibrosteatotest (C) can be used to screen the stage of the disease requiring medical therapy (stage 2 or more of fibrosis and steatosis of the liver). The determination of the stage of fibrosis and steatosis of the second and more stages determines the group of patients of NAPP, for which drug therapy should be performed in connection with a greater likelihood of progressing fibrosis. To assess the information content of the model created, a comparative analysis was carried out with GUCI, APRI, Fib4, Forns, MDA. The results of the AUROC analysis are presented in Figure 3. Available for comparative analysis of models of steatosis of the liver was not found.

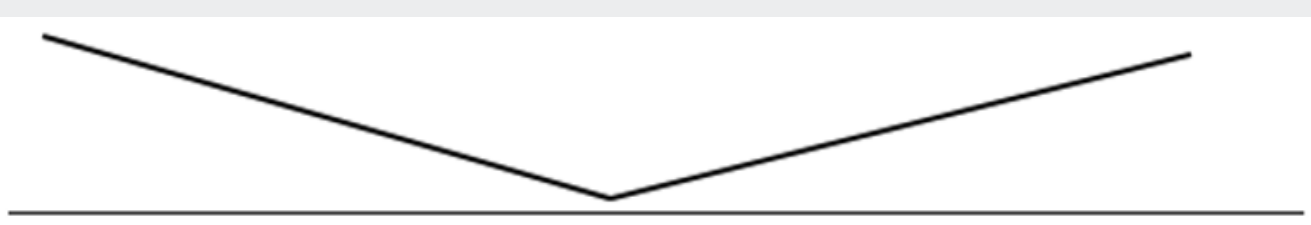

Neat steatosis or steatosis 1 grades

Stage of Liver Stematosis $(S)=-15.612+0.046$ Years (Years) -1.905x (Diabetes Mellitus-1, Absence-0) + 0.48x Erythrocytes + 0.134xbright Bilirubin (Mmol / L) + 1,043 halbumin (g \ dl) +) + 0,148xcpб (Unit) 0,022xa-tinin (Mkmol \Л) + 0,061xthe weight (Kg) - The Constant 15,612

Sensitivity of the Fibrosteatotest model (C) $88 \%$

Specificity of the Fibrosteatotest model @ $83 \%$

Positive predictive ability $88 \%$

False positive response $11 \%$

False negative response $13 \%$

Figure 1: Discriminant equation for the diagnosis of hepatic steatosis of the second and more stage.

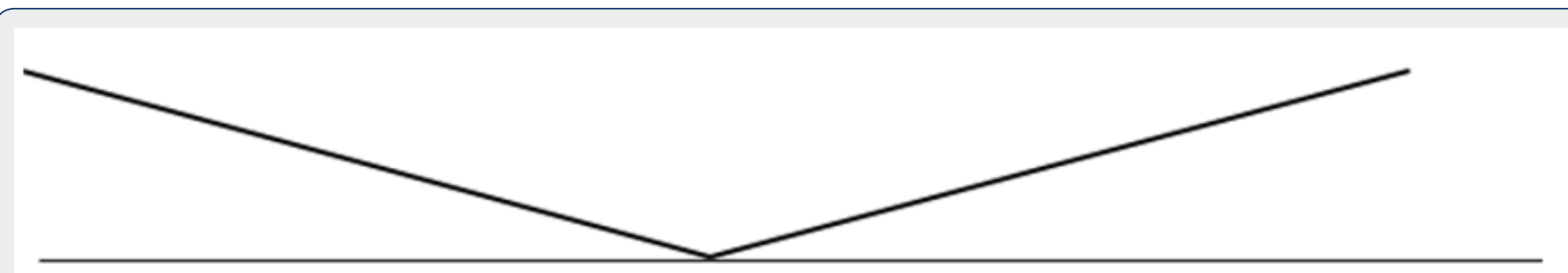

No fibrosis or fibrosis 1 degree

0.074

Fibrosis 2 degrees and higher

Stage of Liver Fibrose $(F)=4,187-2,4 x$ (IHD-presence-1, absence-0) + 0,026xACT (unit / liter) -0,21xGeneral bilirubin ( $\mu$ mol / l) + 0,316xDirect bilirubin $(\mu \mathrm{mol} / \mathrm{l})-0,105$ total protein $(\mathrm{r} \backslash \mathrm{dL})+0.016 x$ Cretinine $(\mathrm{mmol} / \mathrm{L})+0.329$ x-glycated hemoglobin $(\mathrm{mmol} / \mathrm{L})+$ Constant 4.187

Sensitivity of the Fibrosteatotest model (c) $70 \%$

Specificity of the Fibrosteatotest model @ $72 \%$

Positive predictive power $71 \%$

False positive response $29 \%$

False negative response $27 \%$

Figure: 2 A discriminatory equation for the diagnosis of liver fibrosis of the second and more stage. 


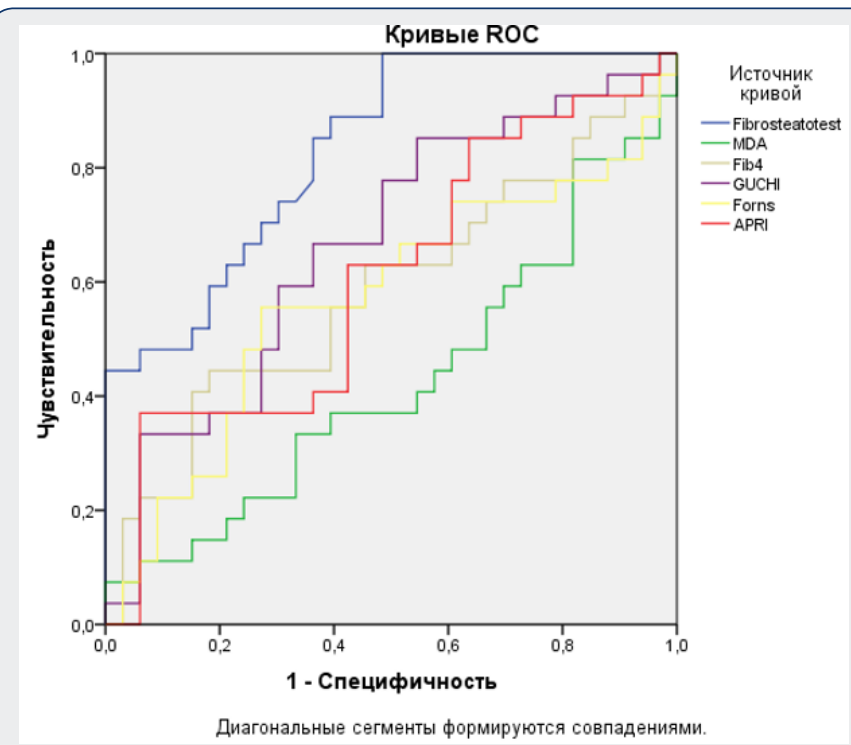

Figure: 3 AUROC-analysis of prognostic value of serum tests of non-invasive evaluation of fibrosis in patients with NAZHBP. Area under the ROC curve: Fibrosteatotest $0.830 \pm 0.029$; GUCI $0.667 \pm 0.041$; APRI $0.607 \pm 0.043$; Fib4 $0.586 \pm 0.044$; Forns $0.570 \pm 0.045$; MDA $0.426 \pm 0.044$.

Table 2: Results of the correlation analysis of the stage of fibrosis and steatosis of the liver with parameters of activity of inflammation, insulin resistance, dyslipidemia.

\begin{tabular}{|c|c|}
\hline $\begin{array}{c}\text { Parameters of liver structure in } \\
\text { NAZHBP }\end{array}$ & $\begin{array}{c}\text { Indicators with correlation } \\
\text { coefficient } \mathbf{r} \geq \mathbf{0 . 3}\end{array}$ \\
\hline Elasticity of the liver - fibrosis, Kpa & $\begin{array}{c}\text { The level of AST, insulin, BMI and } \\
\text { its deviation from the norm, the } \\
\text { stage of steatosis of the liver }\end{array}$ \\
\hline Steatosis of the liver, $\mathrm{dB}$ & $\begin{array}{c}\text { The level of leukocytes, CRP, } \\
\text { cholesterol, HDL, the coefficient of } \\
\text { atherogenicity, triglycerides, BMI } \\
\text { and its deviation from the norm }\end{array}$ \\
\hline
\end{tabular}

Table 3: Results of discriminant analysis for the diagnosis of hepatic steatosis in the second and more stages in patients with NUZHBP (Fibrosteatotest (C).

\begin{tabular}{|c|c|}
\hline $\begin{array}{c}\text { Parameters determining hepatic } \\
\text { steatosis } \geq 2 \text { stage }\end{array}$ & $\begin{array}{c}\text { Weight coefficients of the } \\
\text { discriminant function }\end{array}$ \\
\hline Age (years) & 0,046 \\
\hline Presence of diabetes mellitus & $-1,905$ \\
\hline Number of erythrocytes, $\mathrm{x} 10 \times 12$ & 0,948 \\
\hline Level of direct bilirubin, $\mu \mathrm{mol} / \mathrm{l}$ & 0,134 \\
\hline Level of albumin, g / dL & 1,043 \\
\hline CRP level, units & 0,148 \\
\hline Creatinine, mmol / l & $-0,022$ \\
\hline Weight, $(\mathrm{kg})$ & 0,061 \\
\hline Constant & $-15,612$ \\
\hline
\end{tabular}

\section{The Discussion of the Results}

Changes in diet and lifestyle (hypodynamia), increased consumption of carbohydrates and fats, alcohol, led to a wide spread of NAZHBP. It is generally accepted that early diagnosis and treatment can prevent the progression of chronic liver disease. Fibrosis and steatosis of the liver 2 stages or more is an indication to the beginning of drug therapy. In our study, we presented the possibilities of noninvasive diagnosis of fibrosis and steatosis of the liver on a Fibro scan Fibro Scan 502 TOUCH apparatus with an ultrasound transducer for obese patients with CAP software. The technique is highly informative, safe in any age and state of the patient, but is inaccessible. Creation of accessible non-invasive methods for diagnosis of the stage of fibrosis and steatosis of the liver in NAZHBP is in demand by clinical practice. Given the high diagnostic accuracy of diagnosis of fibrosis and fibroscans in fibroscan (more than 90\%) compared to liver biopsy, it is justified to create mathematical models for diagnosis of fibrosis and steatosis of the liver using its data as a standard instead of liver biopsy. To create models for the diagnosis of steatosis and fibrosis in stages, more patients are needed. For practice, it remains urgent to identify the group of patients who need to start therapy (fibrosis and steatosis more than 2 stages - the Fibrosteatotest (C) test solves this problem), since it has a high risk of progression not only to liver disease, but also the realization of cardiovascular risks and development diabetes mellitus. As can be seen from the formula of the created model, in the progression of the disease, the growth of the stage of fibrosis and steatosis, the weight of the patient, the growth of the stigma of insulin resistance, and the activity of inflammation are important.

An important point in the treatment of NAJBP is the identification of patients who have advanced stages of fibrosis and steatosis of the liver with a risk not only of liver failure, but also of metabolic disorders. Timely and high-quality therapy of NAJBP in this category of patients with mandatory monitoring of the stage of fibrosis and steatosis of the liver is important for prognosis. Therefore, the development and application of available noninvasive methods for the diagnosis of fibrosis and steatosis of the liver remains one of the priority areas in hepatology. The proposed diagnosis model Fibrosteatotest (C) is a simple and affordable method for the clinician.

\section{References}

1. Ing Khan, Tsung Ping Shi, An Lin Ma Yong Xu, Xiao Dong Ding, Jian Gao Fan (2014) A randomized, vitamin E-controlled trial of Bycyclol with metformin in patients with non-alcoholic fatty liver disease (NABE) with impaired fasting glycemia. Clin Drug Investig 34: 1-7

2. MC Semenistaya, Fang Jiangao, OV Velichenko, EA Kuznetsova, Ch S Pavlov (2017) Nonalcoholic fatty liver disease: a comparative assessment of approaches to diagnosis and therapy in the Russian Federation and the People's Republic of China. Rosh Gastroenterol, Hepatol Coloproctolum 27(6): 63-70. 
(C) (P) This work is licensed under Creative

To Submit Your Article Click Here: Submit Article

DOI: $10.32474 /$ CTGH.2018.01.000111

$\begin{gathered}\text { Current Trends in Gastroenterology } \\ \text { and Hepatology }\end{gathered}$
Assets of Publishing with us
- Global archiving of articles
- Immediate, unrestricted online access
- Rigorous Peer Review Process
- Authors Retain Copyrights
- Unique DoI for all articles

